\title{
A Predictive Model of Multipath Effect Contribution to GNSS Positioning Error for GNSS-based Applications in Transport and Telecommunications
}

\author{
Darko Špoljar ${ }^{1,2}$, Ivan Štajduhar ${ }^{1}$, Kristijan Lenac ${ }^{1}$ and Renato Filjar ${ }^{1,2^{*}}$ \\ ${ }^{1}$ Faculty of Engineering, University of Rijeka, Rijeka, Croatia \\ ${ }^{2}$ Krapina University of Applied Sciences, Krapina, Croatia \\ *E-mail of corresponding author: renato.filjar@gmail.com
}

\begin{abstract}
Global Navigation Satellite System (GNSS)-based applications rely on the quality of the GNSS position, navigation, and timing (PNT) services, accomplished through measurement and processing of satellite signals propagation characteristics in a process commonly known as satellite navigation. GNSS positioning performance is in the foundation of the quality of service of GNSS-based applications including the growing number of them in transport, traffic and Intelligent Transport Systems segments, thus a need for a common and independent approach. Here, we propose a novel method for the assessment of the contribution of a single cause to the over-all GNSS positioning error. Proposed method is demonstrated in the case of the GNSS multipath effects, resulting with the experimental predictive model of the direct multipath contribution to GNSS positioning error. The predictive models developed in this research is aimed at deployment in the GNSS positioning performance assessment for GNSS-based applications in transport and telecommunications.
\end{abstract}

Received 18 November 2021

Accepted 6 December 2021

Published 22 December 2021

Keywords: GNSS, positioning performance, multipath, statistical modelling

\section{Introduction}

Modern transport and telecommunication systems and services rely increasingly upon the utilisation of Positioning, Navigation, and Timing (PNT) services provided by Global Navigation Satellite Systems (GNSS). The quality of the GNSS PNT services is embedded deeply in the operations and the quality of mobile communications networks, the internet, Intelligent Transport Systems, co-operative and autonomous vehicles, and the transport logistics, to name the most representative segments. The understanding of the adverse causes and their effects on the GNSS positioning performance in the transport and telecommunications operational scenarios makes both the core GNSS and the GNSS-based applications more robust and reliable.

Here we contribute to the subject with a study of the GNSS multipath effects as one of the major contributors to the GNSS positioning performance degradation in scenarios of transport and mobility-related GNSS applications. We propose a novel generalised method for the assessment of the single (here: multipath) effect contribution to the GNSS positioning error budget, and demonstrate its feasibility in an experimental scenario of potential GNSS utilisation in transport. As the result of the proposed methodological approach, we develop two competing experimental predictive models of the multipath-caused GNSS positioning error, and decide the

(C) The Author(s) 2021. Published by CIEES Association Press. This work is licensed under a Creative Commons Attribution-NonCommercialNoDerivs 4.0 Generic License. winner based on the statsitics-based performance (quality of prediction) assessment.

\section{Method}

The formal GNSS positioning error model and the generalised experimental method are proposed here for the determination of the contribution of the adverse multipath effects to the over-all GNSS positioning performance degradation in the real GNSS positioning environment. The method formulation and formalisation is founded on the practical research results reported for particular GNSS positioning environment cases in [1], [2], [3], and [4]. The method relies on utilisation of the satellite navigation SDR [5], [6] receiver for postprocessing of the raw GNSS pseudorange observations that bear marks of specific positioning environment effects: exposure to ionospheric, tropospheric, or multipath effects, or to GNSS systemic problems (incorrect broadcast satellite ephemeris, satellite failures, atomic clock issues etc.) [2,7].

An additive GNSS positioning performance model is assumed in the generalised form of (1) [8].

$$
\vec{p}_{e}(t)=\vec{x}(t)+\vec{\epsilon}(t),
$$

with:

$$
\vec{p}_{e}(t)=x_{e}(t) \cdot \vec{i}+y_{e}(t) \cdot \vec{j}+z_{e}(t) \cdot \vec{k} \quad \text { GNSS receiver }
$$

estimated position in WGS-84 reference frame at the time of positioning sampling $t$.

$\vec{x}(t)=x_{0}(t) \cdot \vec{\imath}+y_{0}(t) \vec{\jmath}+z_{0}(t) \vec{k} \quad$ GNSS receiver correct (true) position in WGS-84 reference frame at the time of positioning sampling $t$. 
GNSS position estimation error vector, due to effects of positioning environment and GNSS systemic problems at the time of positioning sampling $t$.

$\vec{\epsilon}(t)$ With the reference to (1), we define the GNSS positioning error model using Definition 1, as given below. Definition 1 utilises the WGS- 84 common GNSS reference co-ordinate system to uniquely define the position of every element in space [7].

Definition 1: GNSS positioning error model is defined as an additive model that comprises contributions of the User Equivalent Range Error sources: the control and space segment error sources atomic clock errors, satellite ephemeris errors, depicted as $\overrightarrow{\epsilon_{c s}}(t)$, the user segment error sources an error due to multipath, $\vec{\epsilon}_{m}(t)$, the error due to receiver internal noise, $\vec{\epsilon}_{r}(t)$, the propagation media error sources (an error due to ionospheric effects, $\vec{\epsilon}_{i}(t)$, and error due to tropospheric effects, $\vec{\epsilon}_{t}(t)$, added to the true position $\vec{x}$ to yield the estimated position $\vec{p}_{e}(t)$, as defined in (1), and (2). The components of the estimated position, true position and error vectors are presented in the WGS-84 reference frame.

$\vec{p}_{e}(t)=\vec{x}(t)+\vec{\epsilon}_{c s}(t)+\vec{\epsilon}_{l}(t)+\vec{\epsilon} t(t)+\vec{\epsilon}_{m}(t)+\vec{\epsilon}_{r}(t)$,

The positioning environment impacts the GNSS position estimation process through the pollution of raw GNSS pseudorange measurements with detrimental effects. We propose the generalised method for estimation of an individual detrimental effect's contribution to GNSS positioning error, using Theorem 1.

Theorem 1: Let us assume a mechanism exists, such that overcomes the one error source selected for consideration $\overrightarrow{\epsilon_{l}}(t)$. Let us assume the post-processing GNSS pseudoranges method arranged with the two scenarios, as follows.

Scenario 1: Individual detrimental effects in GNSS pseudorange observations remain uncorrected, while the other sources of GNSS pseudorange measurement errors are processed at will (either uncorrected, or corrected with related correction models). Scenario 1 produces a time series of the position estimates $\vec{p}_{e, u c}(t)$.

Scenario 2: Individual detrimental effects in GNSS pseudorange observations are corrected, while the other sources of GNSS pseudorange measurement errors are processed at will (either uncorrected, or corrected with related correction models) in the same manner as in Scenario 1. Scenario 2 produces a time series of position estimates $\vec{p}_{e, c}(t)$.

The contribution of the selected individual detrimental source to the over-all GNSS positioning error under the real conditions of the positioning environment may be determined as the difference between the outcomes of Scenarios 1 and 2, respectively, as noted in (3).

$$
\begin{aligned}
\vec{\epsilon}_{l}(t) & =\vec{p}_{e, u c}(t)-\vec{p}_{e, c}(t)= \\
& =\epsilon_{l, x}(t) \cdot \vec{i}+\epsilon_{l, y}(t) \cdot \vec{j}+\epsilon_{l, k}(t) \cdot \vec{k}
\end{aligned}
$$

Proof: The selection of the appropriate mechanism to overcome the targeted detrimental effect depends on the nature of the error source. Ionospheric effects may be mitigated using a single or a combination of the following approaches [2, 7]: (i) deployment of standard (Klobuchar or NeQuick) ionospheric delay corrections, (ii) utilisation of dual-frequency processing that removes the ionospheric effects almost entirely, (iii) application of post-processed TEC maps for the determination of GNSS ionospheric correction, or (iv) application of more accurate GNSS ionospheric delay models maintained and operated by trusted sources. Tropospheric effects may be mitigated using a selection of a suitable GNSS tropospheric delay correction model, such as [7]: Saastamoinen, based on the actual weather conditions, Saastamoinen, based on the standard atmosphere, Hopfield, Niell. Multipath effects [9] may be mitigated through the deployment of two independent GNSS pseudorange data sets, one taken under multipath exposed conditions as Scenario 1 data, and the other collected geographically close to the previous one, but without the effects of multipath (additional mitigation through GNSS receiver-specific signal processing, or multipathsuppression aerials). Satellite ephemeris and atomic clock errors [7] may be overcome through utilisation of the actual data on satellite orbits and atomic clock performance, such as those regularly maintained by the International GNSS Service (IGS). Distillation of the selected detrimental effect's contribution to the over-all GNSS positioning error using (3) may be proven by differentiating, as stated in (3), using the respective forms of (2) for $\vec{p}_{e, u c}(t)$ and $\vec{p}_{e, c}(t)$.

A method for the GNSS positioning performance assessment established in Definition 1 and in Theorem 1 is depicted in Figure 1. It is formalised and generalised from a series of methodologies applied to the experimental assessment of the GNSS positioning performance in related positioning environments, accentuating the effects of targeted sources of GNSS positioning degradation: GNSS spoofing [1], space weather and ionospheric [2, 4], tropospheric [3], and multipath [10] effects.

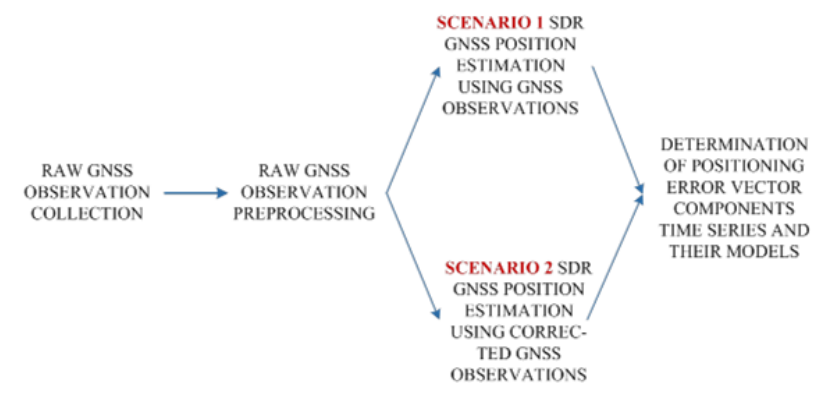

Figure 1. Method for the GNSS positioning performance assessment.

The GNSS positioning performance assessment method comprises the four essential task groups (Figure 1). Firstly, a GNSS receiver of the appropriate grade and purpose is utilised in the positioning environment under scrutiny to collect raw GNSS observations (pseudoranges and the observed navigation messages), while storing data locally. Raw GNSS observations are to be preprocessed to transform to the format suitable for further process, to ensure utilisation of observations taken at the 
same time instant in both Theorem 1 Scenarios, and to correct for the individual effects using either standard, or bespoke correction models. The same correction models should be deployed in both scenarios of Theorem 1 . Corrected GNSS observations feed the Software-Defined Radio (SDR) [5, 6] satellite navigation receiver in the post-processing mode in the third task group of the method.

The two scenarios of Theorem $\mathbf{1}$ are emulated through configuration of the SDR satellite navigation receiver, providing flexibility to emulate a range of market-ready receiver classes and complete control over the selection of a particular individual GNSS positioning performance degradation source. The same satellite position estimation method should be utilised for both scenarios of Theorem1.

The position-estimation process yields two time series of satellite position samples: one with all the sources of positioning errors suppressed by utilisation of correction models, and the other with the retained effects of the selected positioning error source. Contribution of a selected positioning error source to the over-all GNSS positioning error is determined using Theorem 1. Presumptions of Theorem 1 ease the requirements for accuracy of correction models. Assuming the additive model, the only requirement imposed is the need to utilise the same correction models for both scenarios of Theorem 1. Providing the consistency, the method proposed here is independent with regard to the correction model, the position estimation algorithm, and the receiver class.

Based on exploratory statistics analysis, including statistical distribution and partial auto-correlation analysis, the candidate forecasting models of the positioning error vector components were developed, using the following methods:

(i) Auto-Regressive (AR) model, of the form (4), and of the order $p$, determined by the statistical properties of the process in examination (i.e. experimental data generated by the process) $[11,12]$ :

$x_{t}=a+\sum_{i=1}^{p} b_{i} \cdot x_{t-i}+\epsilon_{t}$.

where:

$x_{t} \ldots$ denotes time series of variable values for which the $\operatorname{AR}(p)$ model is developed

$a \ldots$ denotes the intercept value

$b_{i}, i=1, \ldots, p \ldots$ denotes $\operatorname{AR}(p)$ model coefficients

$p$... denotes the order of the $\operatorname{AR}(p)$ model

$\varepsilon_{t} \ldots$ denotes time series of white noise samples

(ii) Exponential Smoothing (ES) model, of the component form (5), as defined by [12], and with related parameters determined by statistical properties of the process in examination (i.e. experimental data generated by the process $)[12,13]$ :

$$
\begin{aligned}
& \hat{y}(t+1 / t)=l(t)(\text { forecast eq. }) \\
& l(t)=\alpha \cdot y(t)+(1-\alpha) \cdot l(t-1)(\text { smoothing eq. })
\end{aligned}
$$

where: $\hat{y}(t+1 \mid t) \ldots$ denotes the estimate of the time series variable value at the time instant $t+1$, given the knowledge of time series at the time instant $t$

$y(t) \ldots$ denotes time series variable value at the time instant $\mathrm{t}$

$l(t) \ldots$ denotes the level (smoothed value) of the time series at the time instant $t$

(iii) Artificial Neural Network (ANN) model, whose architecture was determined by the statistical properties of the process under examination [13].

The model development methodology thus extends the experimental data-based statistical learning nature. Model validation may be performed using the common statistical tests applied on the model residuals [13].

\section{Research results and discussion}

The method proposed in Section 2 is demonstrated in the case of identification of the multipath contribution to the over-all GPS positioning error, based on an experiment set up in a rural environment (Kalinovac, northern Croatia). The aim of the experiment was to identify the potential multipath effect caused by vegetation. We utilised Theorem 1, with the noted restriction of the standard multipath correction model, which is still not readily available for mass-market GNSS units. The problem was overcome through the experiment design that involved two sources of raw GNSS observations (Figure 2). One source collected raw GNSS observations from the intensive multipath positioning environment we allow it intentionally. The other one was placed close to the first one, but in the environment cleared of any multipath effect. Established that way, the two raw GNSS observation sources were exposed to the same level of all the other GNSS positioning error effects except the multipath, thus creating a multipath-controlled positioning environment.

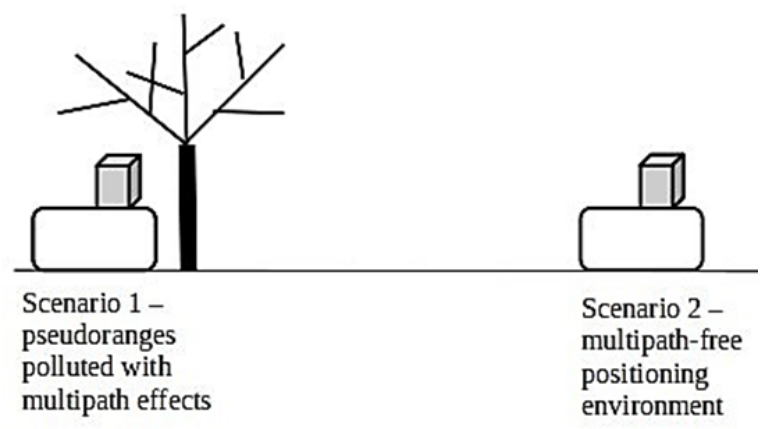

Figure 2. Experimental realisation of the Theorem 1 scenarios.

Two commercial-grade single-frequency satellite navigation receivers in Huawei Mate Lite smartphones were used, one of them collecting the GPS pseudorange observations behind a cherry tree, thus creating a vegetation-related multipath environment [9], and the other one being set in the multipath-free and clear-sky visibility conditions in the close vicinity of the first satellite navigation receiver. Duration of the experiment was $30 \mathrm{~min}$, having $1 \mathrm{~s}$ pseudorange sampling rate, thus resembling the conditions of targeted GNSS based applications in transport and telecommunications. The design of the experiment was performed in conceptual sense of the method proposed in Section 2, with a slight 
modification involving the utilisation of two GNSS receivers to overcome the problem of non-existing general multipath correction model, as a presumption of Theorem 1.

The GPS pseudoranges were collected and stored on smartphones using the Android application Geo++ RINEX Logger [14], taking the advantage of the Android Location API feature that allows for direct raw GPS pseudorange collection [15]. RTKLIB, an open-source SDR satellite navigation receiver [16], was utilised using the suitable commercial-grade single-frequency GPSonly receiver configuration. The standard ionospheric (Klobuchar), tropospheric (standard atmosphere-based Saastamoinen) and satellite clock error models were deployed in research. Pre-processing and statistical analysis of multipath contribution to GPS positioning performance according to the Theorem 1 method were conducted with bespoke software developed by our team in the open-source $\mathrm{R}$ environment for statistical computing [17].

Time series of the multipath contribution to the overall GPS positioning error was split into the WGS-84 vector components in northing, easting, and vertical directions, respectively, and depicted in Figure 3.

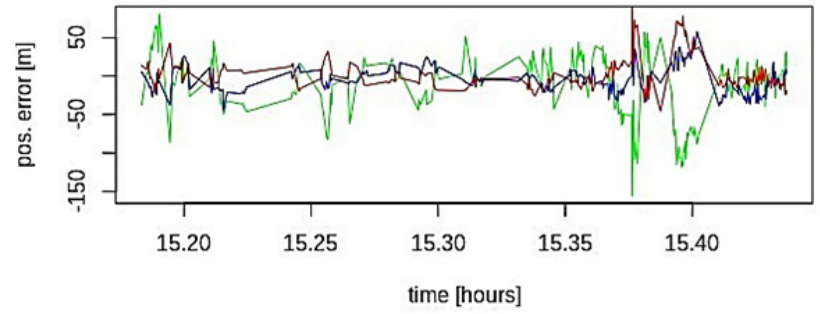

Figure 3. Time series of northing (red), easting (blue), and vertical (green) components of the multipath contribution to the GPS positioning error in the experiment conducted.

Results of exploratory statistical analysis of the multipath contribution to the GPS positioning error are summarised in Figures 4 and 5.

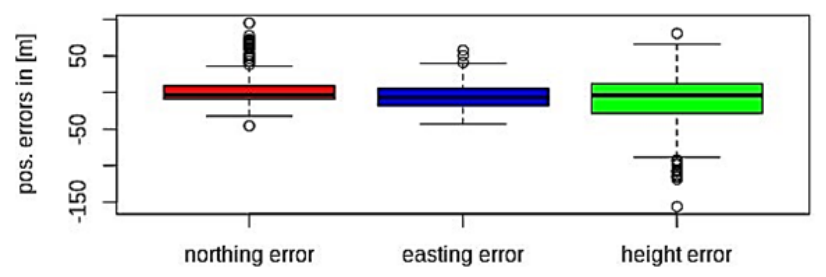

Figure 4. Box-plot diagrams of the northing (left), easting (centre), and vertical (right) components of the multipath contribution to the GPS positioning error in the experiment conducted.
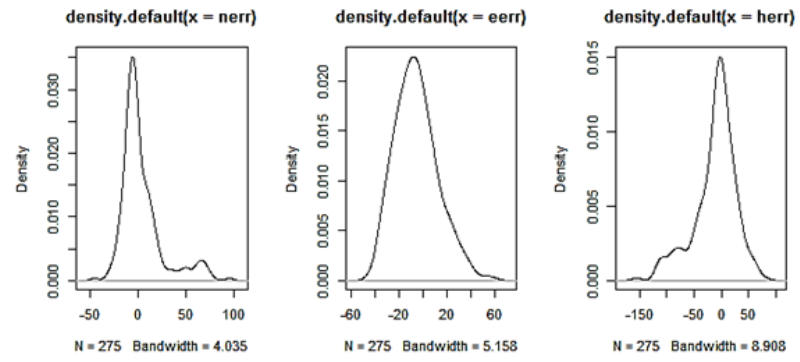

Figure 5. Estimates of experimental statitical distributions of northing (nerr, left), easting (eer, centre), and vertical (height, right) components of the multipath contribution to the GPS positioning error in the experiment conducted.

Easting error extends the most stable behaviour, having just a handful of outliers, and an only slightly skewed Gaussian-like statistical distribution. Northing error extends the most variable dynamics, exhibiting nonGaussian distribution of the northing error time series. Vertical error both biased and variable behaviour, although its statistical distribution does not depart from normal one extensively.

Exploratory statistical analysis provides the foundation for a decision on error model class selection. Multipath causes non-linear positioning error time series, thus calling for selection of non-linear and stochastic models for multipath GPS positioning error modelling [18]. We examined the time series of the respective components of multipath contribution to GPS positioning error for their partial auto-correlation functions (PACFs) (Figure 6).
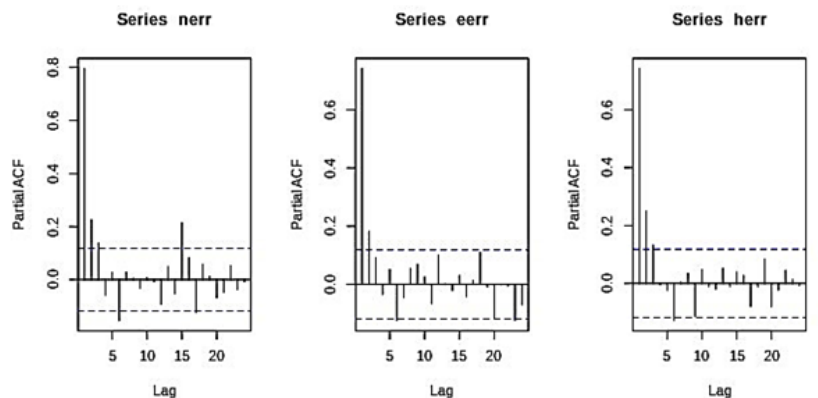

Figure 6. PACF diagrams of northing (nerr, left), easting (eer, centre), and vertical (height, right) components of the multipath contribution to the GPS positioning error in the experiment conducted.

\subsection{Auto-regressive (AR) forecasting models}

The Auto-Regressive (AR) forecasting models of multipath error contribution to total GPS positioning error were developed based on experimental multipathpolluted GPS observations, using the methodology presented in Section 2. The PACF assessment serves as the rule of thumb for the determination of the order of the Auto-Regression (AR) Model. The PACF assessment confirmed the need for complex models of the all GPS positioning error vector components [11]. The northing error component required the most complex model, with its remotest statistically significant component falling within the $(1,15)$ lag region emerging at the lag 15 .

In demonstration of PACF analysis results deployment for model development of the multipath contribution to the over-all GPS positioning error, the Auto-Regressive (AR) AR( $p)$ models of the order $\mathrm{p}$ of components of the multipath contribution to GPS positioning error were developed, in the form given in (4) [11].

AR models were developed for multipath contribution to GPS positioning error components in northing, easting, and vertical directions, of the order of 15,6 , and 6 , respectively. 


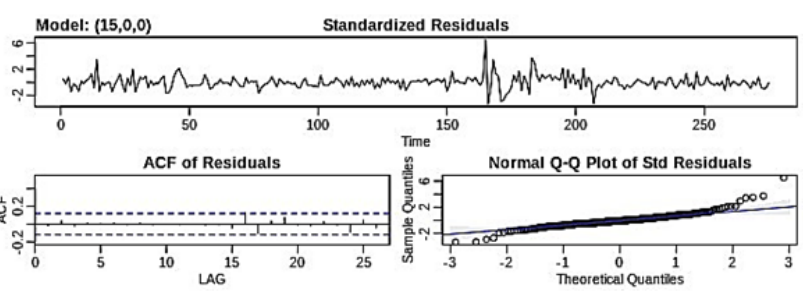

Figure 7. The experimental $A R_{\text {northing }}(6)$ model of the northing multipath contribution to the over-all GPS positioning error.

The R-package astsa [19] features a suitable function sarima used in the bespoke developed $\mathrm{R}$ software that returns details of the experimental data-based $A R_{\text {northing }}(15), \quad A R_{\text {easting }}(6)$, and $A R_{\text {vertical }}(6)$ models descriptions and validation results, depicted in Figures 7 , 8 , and 9 , respectively.

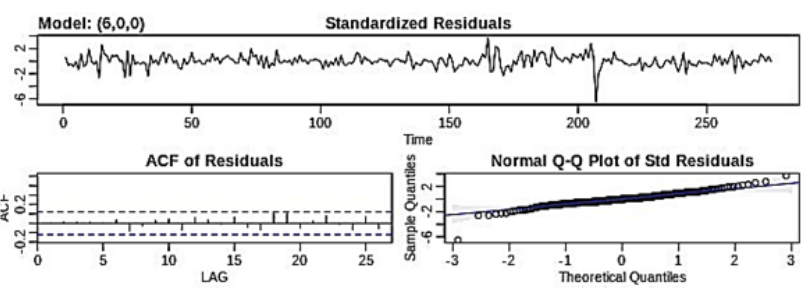

Figure 8. The experimental $A R_{\text {easting }}(2)$ model of the easting multipath contribution to the over-all GPS positioning error.

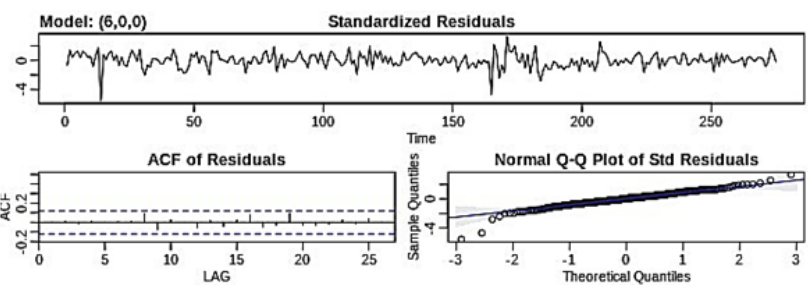

Figure 9. The experimental $A R_{\text {vertical }}(6)$ model of the vertical multipath contribution to the over-all GPS positioning error.

The $\operatorname{AR}(p)$ models analysis reveals a lack of statistically significant components of the respected autocorrelation functions in all three cases considered. The room for model refinements exists still, because the respected Q-Q plots reveal a detachment from the ideal line curve on both tails of the related statistical distributions.

\subsection{Exponential-Smoothing (ES) forecasting models}

The Exponential-Smoothing (ES) forecasting models of multipath error contribution to the total GPS positioning error were developed based on experimental multipath-polluted GPS observations using the methodology presented in Section 2. Model development process yields the related model parameters, as given in Table 1.

Table 1. ES forecasting model parameters for related multipath contribution to the total GPS positioning error components.

\begin{tabular}{cccc}
\hline & Northing error & Easting error & Vertical error \\
\hline$\alpha$ & 0.6182 & 0.6419 & 0.5706 \\
$l(0)$ & 8.7381 & 6.2098 & -6.3881 \\
\hline
\end{tabular}

The ES models near-term forecasts (20 samples after the 255 samples-long training section) of northing, easting, and vertical multipath contribution to total GPS positioning error are presented in Figures 10 - 12., respectively, along with experimental observations for comparison. Combined Q-Q diagrams of related models are depicted in Figure 13. Q-Q diagrams of the northing and easting multipath contribution models reveal a rather large distraction from theoretical normal distribution, and a general failure in their ability to model the multipathrelated contribution to GPS positioning error. Vertical multipath contribution was modelled slightly better, as revealed by the related model's Q-Q plot.

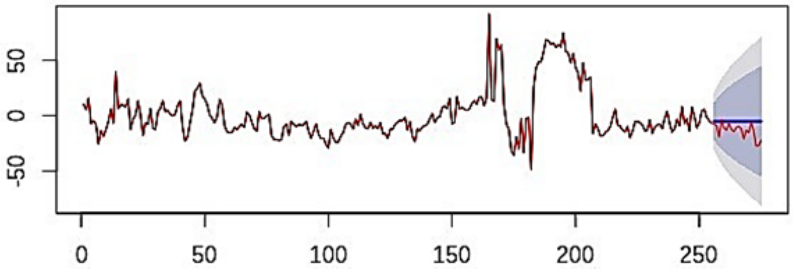

Figure 10. Northing error time series training set (255 samples) and the exponential smoothing-based 20 samples estimates.

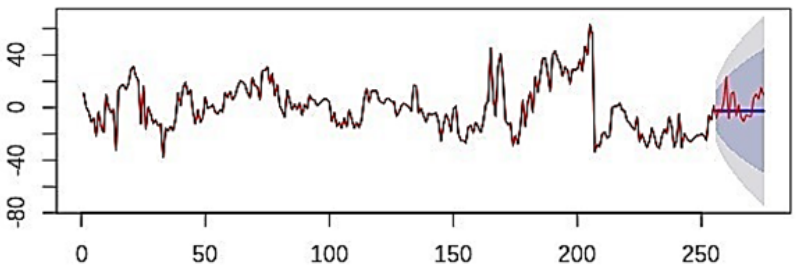

Figure 11. Easting error time series training set $(255$ samples) and the exponential smoothing-based 20 samples estimates.

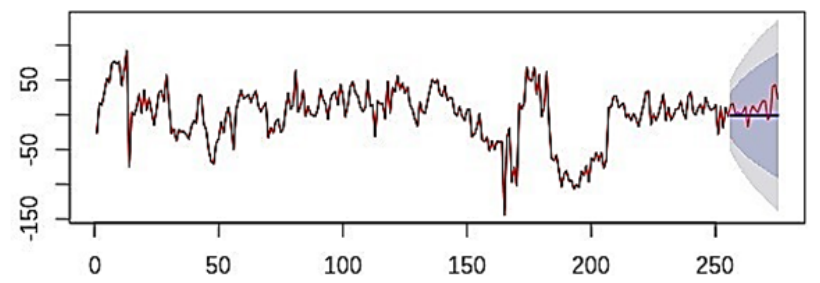

Figure 12. Vertical error time series training set (255 samples) and the exponential smoothing-based 20 samples estimates.

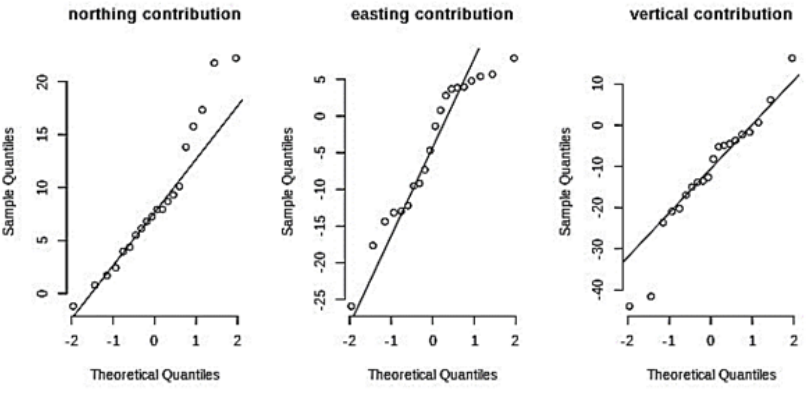

Figure 13. Combined Q-Q diagrams of the models of the ES modelled northing (left), easting (centre), and vertical (right) multipath contribution to the total GPS positioning error. 


\subsection{Artificial neural network forecasting models}

Artificial neural network forecasting models of multipath error contribution towards the total GPS positioning error were developed based on experimental multipath-polluted GPS observations using the artificial neural network methodology presented in Section 2. In particular, we took the self-adaptive ('automated') approach in scaling the artificial neural network proposed by [12] and deployed in the forecast library of the $\mathrm{R}$ environment for statistical computing. Model development process yields the related model parameters, as given in Table 2 .

Table 2. ANN model parameters for related components of the multipath contribution to total GPS positioning error.

\begin{tabular}{cccc}
\hline & $\begin{array}{c}\text { Northing } \\
\text { contribution }\end{array}$ & $\begin{array}{c}\text { Easting } \\
\text { contribution }\end{array}$ & $\begin{array}{c}\text { Vertical } \\
\text { contribution }\end{array}$ \\
\hline $\begin{array}{c}\text { Number of } \\
\text { neural } \\
\text { networks } \\
\text { averaged } \\
\text { Network }\end{array}$ & 20 & 20 & 20 \\
$\begin{array}{c}\text { structure (input } \\
\text { neurons - }\end{array}$ & $17-9-1$ & $2-2-1$ & $3-2-1$ \\
$\begin{array}{c}\text { hidden neurons } \\
\text { - output } \\
\text { neurons) }\end{array}$ & & & \\
$\begin{array}{c}\text { Number of } \\
\text { weights } \\
\text { Estimated } \\
\text { standard } \\
\text { deviation }\end{array}$ & 172 & 9 & 11 \\
\hline
\end{tabular}

The performance analysis revealed a poor performance of the easting component model, a slightly better one for the northing, and the best performance for the vertical component model. In general, the ANN approach under-performed, compared against the adequate $\operatorname{AR}(p)$ models. Further consideration leads to the conclusion of a sparse data set required for the ANN training, advising for future research with a larger data set.

Forecasts from NNAR(17,9)

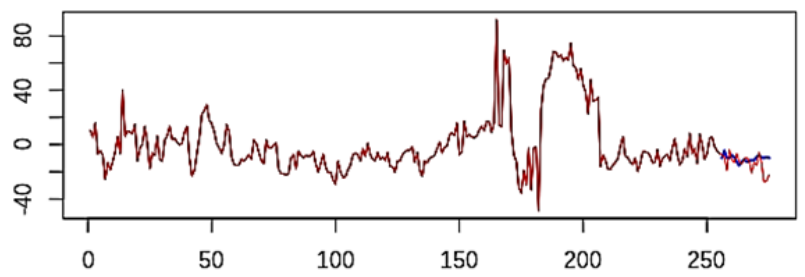

Figure 14. Northing error time series training set (255 samples) and the neural network-based 20 samples estimates.

Forecasts from NNAR(2,2)

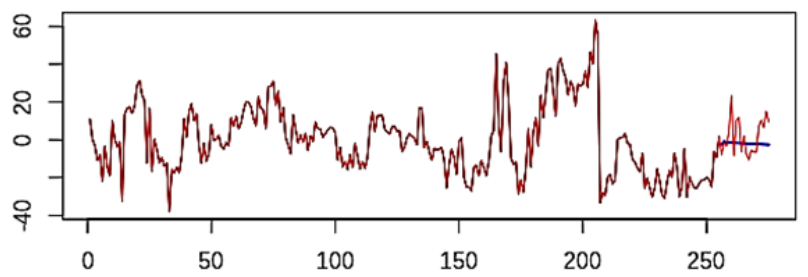

Figure 15. Easting error time series training set $(255$ samples) and the neural network-based 20 samples estimates.

\section{Forecasts from NNAR(3,2)}

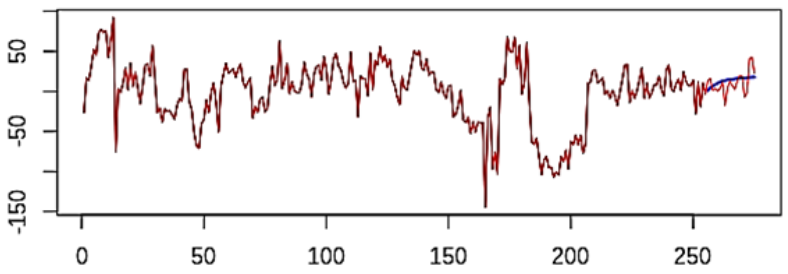

Figure 16. Vertical error time series training set (255 samples) and the neural network-based 20 samples estimates.
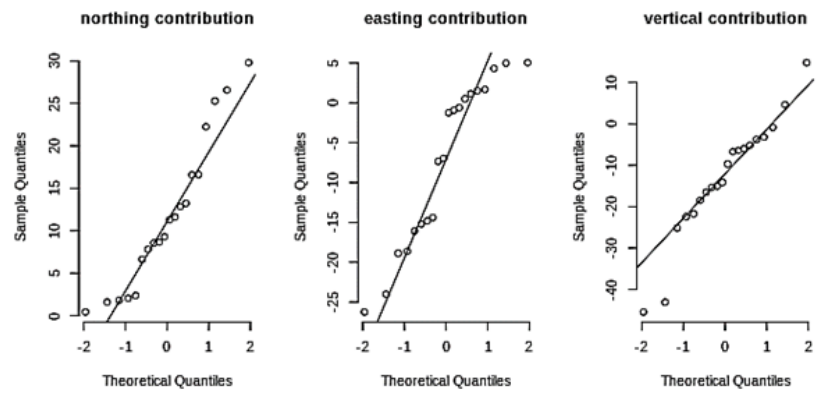

Figure 17. Combined Q-Q diagrams of the models of the ANN modelled northing (left), easting (centre), and vertical (right) multipath contribution to the total GPS positioning error, respectively.

\section{Conclusion}

In the research presented here, we contributed to the problem of the GNSS positioning performance estimation in a given positioning environment and for a given class of applications through the introduction of a novel method for estimating the contribution of a selected source of the GNSS performance degradation. The proposed method relies on the assumption of the additive model of GNSS positioning errors, and utilises experimental GNSS observations (raw pseudoranges and navigation messages) and a configurable SDR satellite navigation receiver. The proposed method was demonstrated on the case of distillation of multipath contribution to the over-all GPS positioning error. Statistical analysis of the multipath contribution was performed by a tailored software we developed in the $\mathrm{R}$ environment for statistical computing.

The proposed method was demonstrated in the case of the assessment of multipath contribution to the total GPS positioning error in the multipath-prone environment. Three different approaches involving statistical learning based on the exploratory statistical analysis of the experimental data were taken in development of nonstochastic forecasting models of multipath contribution to the total GPS positioning error in a given multipathexposing environment, having inadequate forecasting performance, yet with the perspective for improvement, especially on larger experimental data sets. Models of multipath contribution to GNSS positioning error developed in this research may serve as a reference model for GNSS-related applications in transport and telecommunications, requiring robust and reliable GNSS 
Positioning, Navigation, and Timing (PNT service quality.

The future research efforts aim at the multi-GNSS positioning performance estimation, and method development for self-tuning error model development using statistical learning methods, utilising the results of multipath statistical characterisation using the method proposed in this manuscript.

\section{References}

[1] Filić, M. (2018). Foundations of GNSS spoofing detection and mitigation with distributed GNSS SDR receiver. TransNav, 12(4), 649-656. doi: 10.12716/1001.12.04.01

[2] Filić, M, and Filjar, R. (2018). Forecasting model of space weather-driven GNSS positioning performance. Lambert Academic Publishing. Riga, Latvia.

[3] Filić, M, and Filjar, R. (2018). A South Pacific CycloneCaused GPS Positioning Error and Its Effects on Remote Island Communities. TransNav, 12(4), 663-670. doi: 10.12716/ 1001.12.04.03

[4] Filic, M, Filjar, R, and Ruotsalainen, L. (2016). An SDRbased Study of Multi-GNSS Positioning Performance During Fast-developing Space Weather Storm. TransNav, 10(3), 395400. doi: $10.12716 / 1001.10 .03 .03$.

[5] Stewart, W. et al. (2017). Software Defined Radio using MatLab \& Simulink and the RTL-SDR (1st ed). University of Strathclyde. Glasgow, UK. Available at: https://strathprints.strath.ac.uk/54340/

[6] Filjar, R, Huljenić, D, and Lenac, K. (2013). Enhancing Performance of GNSS Position Estimation by Cloud-based GNSS SDR Receiver Architecture Utilisation. Proc of 55th International Symposium ELMAR-2013, 315-318. Zadar, Croatia.

[7] GPS Directorate. (2019). NAVSTAR GPS Space Segment/Navigation User Segment Interfaces (IS-GPS-200K) Interface Specification. GPS Directorate. Washington, DC.
Available at: https://www.gps.gov./technical/icwg/IS-GPS200K.pdf

[8] Gustafsson, F. (2010). Statistical Sensor Fusion (2nd ed). Studentlitteratur. Linköpping, Sweden.

[9] Hannah, B M. (2001). Modeling and simulation of GPS multipath propagation ( $\mathrm{PhD}$ thesis). Queensland University of Technology. Brisbane, QLD.

[10] Rumora, I, Sikirica, N, and Filjar, R. (2018). An Experimental Identification of Multipath Effect in GPS Positioning Error. Transnav, 12(1), 29-32. doi: 10.12716/1001.12.01.02

[11] Woodward, W A, Gray, H L, and Elliott, A C. (2017). Applied Time Series Analysis with R. CRC Press/Taylor and Francis. Boca Raton, FL.

[12] Hyndman, R J, and Athanasopoulos, G. (2021). Forecasting: Principles and Practice (3rd ed). Otexts. Melbourne, VIC. Available at: https://otexts.com/fpp3/

[13] Murphy, K P. (2012): Machine Learning: A probabilistic approach. MIT Press. Cambridge, MA.

[14] Geo++. (2019). Geo++ RINEX Logger. Android application. Geo++ GmbH. Garbsen, Germany.

[15] GSA GNSS Raw Measurements Task Force. (2017). Using GNSS raw measurements on Android devices (white paper). European GNSS Agency. Prague, Czech. Available at: http://bit.ly/2BiQ4s2

[16] Takasu, T. (2013). RTKLIB: An Open Source Program Package for GNSS Positioning (software and documentation). Available at: http://www.rtklib.com.

[17] R Development Core Team (2019). R: A language and environment for statistical computing. $\mathrm{R}$ Foundation for Statistical Computing, Vienna, Austria. ISBN 3-900051-07-0. Available at: http://www.R-project.org.

[18] Huffaker, R, Bittelli, M, and Rosa, R. (2017). Nonlinear Time Series Analysis with R. Oxford University Press. Oxford, UK.

[19] Stoffer, D. (2019). astsa: Applied Statistical Time Series Analysis. University of Pittsburgh. Pittsburgh, PA. Available at: https://cran.r-project.org/web/packages/astsa/index.html 